\title{
70. ON THE PROBLEM OF THE ORIGIN OF COMETS
}

\author{
J. M. WITKOWSK I \\ University Observatory, Poznań, Poland
}

\begin{abstract}
The various hypotheses on the origin of comets are surveyed, with particular reference to the phenomenon of comet-streaming, as revealed by statistical analysis of the directions of the perihelia of cometary orbits. The difficulties of reconciling comet-streaming with any of the hypotheses of cometary origin is pointed out. It is shown that in the case of the interstellar hypothesis these difficulties may be overcome by supposing that the velocities of incoming comets are decreased through the accretion of material from an Oort cloud of solar-system comets. An estimate is made of the total number of interstellar comets in the Galaxy.
\end{abstract}

Nowadays there exists a variety of hypotheses on the origin of comets. From a general point of view the hypotheses may be subdivided into two main groups: those presuming the interstellar origin of comets and those that suppose that comets originate in the solar system.

At the head of the first group is the Laplace hypothesis. Proceeding from the assumption of Kepler and Herschel about the availability of comets in interstellar space, Laplace determined the relative probabilities for comets with elliptical and hyperbolic orbits versus their velocities relative to the Sun. He assumed the radius of the Sun's sphere of action to be $100000 \mathrm{AU}$ and considered the relative velocities at which the comets enter that sphere all to be equally probable. He concluded that there would be a prevalence of elliptical and parabolic orbits, which was in agreement with the statistical data then available.

Laplace's statement of the problem itself has been repeatedly subjected to criticism. Schiaparelli and Niessl considered that proper account must be taken of the Sun's motion in space. Seeliger criticized the assumed velocity interval (zero to infinity). The investigations by Fabry, Moisseev, and Bobrovnikoff ascertained that the frequency of elliptical, parabolic, and hyperbolic orbits depends on the character of the velocity distribution function as well as on the Sun's motion (Hoffmeister, 1951, 1952). As a general result these investigations lead to the conclusion that the orbits of comets cannot provide an answer to the question of the origin of comets.

Not all the arguments put forward against the hypothesis of Laplace are fortunate. It was not without reason that Laplace considered only the velocities of comets relative to the Sun, neglecting the problem of the Sun's spatial motion. Not all authors have stated the problem of the character of the orbits in a sufficiently lucid way. The only possible orbits are ellipses and hyperbolas, since the statistical probability of circles and parabolas (as well as straight lines) is geometrically and dynamically infinitesimally small and need not be taken into consideration.

In the aforementioned hypotheses the a priori existence of ready-made comets in space is accepted. The hypothesis of Lyttleton (1948) differs from the preceding ones in that it considers the process of the passage of the Sun through a uniform interstellar cloud of dust (Bondi and Hoyle, 1944). The particles in that cloud are constrained by 
solar attraction to describe hyperbolas with the Sun as focus. These hyperbolas intersect along a line parallel to the relative velocity vector of the Sun and cloud and which passes through the Sun. Along this line accretion takes place, condensations being formed that according to Lyttleton represent newly formed comets. The hypothesis may be generalized (Gething, 1951; McCrea, 1953) by accepting a nonuniform structure for the cloud.

Lyttleton's hypothesis endeavours not only to explain the origin of comets, but also to give a physical model of cometary structure. While Lyttleton has in general terms succeeded in solving this problem, his hypothesis does not answer the questions raised by critics, and his model of cometary structure disagrees with observations (Richter, 1963).

A quite different way of solving the problem of the origin of comets is based upon the spatial distribution of the major axes of cometary orbits. This approach to the problem will be discussed later. Meanwhile let us turn to the hypotheses that presuppose that comets belong to the solar system. The first such hypothesis, advanced by Lagrange, was supplemented by Proctor and Tisserand, and at present is most extensively developed by Vsekhsvyatskij $(1930,1931,1952)$. In accordance with this outlook comets are the products of volcanic eruptions upon the surfaces of the major planets or their satellites. However tempting this hypothesis may appear at first sight, it encounters considerable difficulties, the principal one being the great initial velocities of eruption needed ( $\sim 67 \mathrm{~km} \mathrm{~s}^{-1}$ for Jupiter), velocities which are unacceptable from the viewpoint of the physics of these planets.

The hypothesis of Oort $(1950,1951)$ belongs to the same group. In general terms, it may be reduced to the following statements. The comets form part of the solar system, for otherwise the percentage of hyperbolic orbits would be considerably greater than is observed. Consequently, the comets accompany the Sun in its motion, i.e., they constitute a cometary cloud. Oort attributes the origin of that cloud to the disintegration of a planet that formerly moved around the Sun between the orbits of Mars and Jupiter; the fragments of this planet gave rise to the formation of both comets and minor planets. Perturbations by Jupiter and other planets caused some of the fragments to be diffused away, and this resulted in the formation of a large cloud of comets situated at present at heliocentric distances between 50000 and $200000 \mathrm{AU}$. Under the disturbing action of neighbouring stars the comets in this 'reservoir' may be either ejected from the solar system or directed back to the Sun, i.e., into the sphere of visibility of the terrestrial observer.

Oort's hypothesis is based to a great extent on the theoretical studies by van Woerkom (1948). However, as Oort remarks, further calculations are necessary. The principal statement in Oort's hypothesis is the denial of the existence of interstellar comets (i.e., the absence of 'original' hyperbolic orbits), as was supposedly proven by Strömgren (1914, 1916), Sinding (1948), and Galibina (1963). The next essential element of this hypothesis refers to the cometary reservoir on the outskirts of the solar system. Orlov (1939) does not believe the cloud could exist, and Lyttleton found in Oort's argument a substantial error in determining the position of the maximum concentration of the major axes of the cometary orbits. 
The general survey of hypotheses considered here brings us to the following conclusions. None of the above-mentioned hypotheses solves the problem of the origin of comets. It should be readily apparent that statistical investigations of orbital forms cannot solve this problem. The existence of hyperbolic orbits suggests only that they have kept their initial character; on the other hand, the orbits of some interstellar comets could have been significantly modified at the edge of the solar system.

Oort's interpretation of the cometary cloud hypothesis, although interesting in itself, is too artificial. Lyttleton's hypothesis is generally more probable, conforming to our knowledge of interstellar space, but it requires more thorough elaboration.

We now turn our attention to a more comprehensive discussion of the problem of the distribution in space of the major axes of cometary orbits and the resulting deductions.

These investigations encounter difficulties because of the yet insufficient empirical data and their complex relationship with the observational conditions. Hoek discussed the matter more than a hundred years ago. Schiaparelli denied that there was any relationship in the distribution of the perihelia of cometary orbits with the galactic equator, whilst Holetschek admitted the existence of such a relationship but attributed it to observational conditions. According to Jantzen, the interpretation by Holetschek is not very convincing.

Svedstrup (1883) investigated the distribution of the perihelia of cometary orbits and pointed out (using a simplified ellipsoidal analysis) their connection with the galactic equator. His work received no response until Oppenheim $(1922,1924)$ again raised this problem. Oppenheim made use of the statistical methods of stellar astronomy, namely, ellipsoidal analysis. He examined separately the distribution of the perihelia of elliptical and parabolic cometary orbits as well as those of the minor planets. The ellipsoid for the minor planets has common features with the ellipsoid for elliptical cometary orbits, both ellipsoids being oriented in the plane of the ecliptic.

A quite different picture is obtained for parabolic cometary orbits. The ellipsoid is directed along the galactic equator, but the differences between the axes are small, from which it may be deduced that cometary material is distributed around the Sun symmetrically. Since the shortest axis is directed towards the vertices of star-streaming Oppenheim concluded that comets must be of interstellar origin. Under the disturbing action of the major planets the interstellar features of cometary orbits are smoothed out in the course of time; this is precisely what has happened in the case of the periodic comets, whose orbits have acquired planetary features, in particular the orientation of their orbital planes to the ecliptic.

About twenty years ago a series of investigations on the subject was initiated at the Poznań University Observatory. The calculations by Svedstrup and Oppenheim have been verified using more extensive statistical material relating to 451 long-period (parabolic) comets (Witkowski, 1953; Hurnik, 1959). It turned out that the results were not greatly influenced by the selection of material, for comets prior to 1700 and after 1900 gave practically identical ellipsoids for the perihelion distribution. This is at variance with the statements of Bourgeois and Cox (1934), who in their extensive studies attributed the results of Oppenheim to the influence of observational conditions. 
It became necessary to check the conclusions by Bourgeois and Cox, all the more now that new studies of the distribution of cometary perihelia have appeared that speak in favour of Oppenheim's results (Tyror, 1957; Kresák, 1957). A critical analysis of the deductions by Bourgeois and Cox was performed at my suggestion by Hurnik (1959) on material embracing the aforementioned 451 comets, because Bourgeois and Cox derived 'Oppenheim's corrected ellipsoid' on the basis only of comets at $\alpha=\omega=90^{\circ}, \omega$ being the argument of perihelion of a cometary orbit and $\alpha$ the longitude of the Earth measured from the ascending node of the cometary orbit. Following the method of Bourgeois and Cox, Hurnik determined anew the 'selection factors' and carried out the calculations varying $\alpha$ and $\omega$ in $45^{\circ}$-steps between 0 and $360^{\circ}$. Hurnik's results demonstrated that the distribution of perihelia was independent of visibility conditions and that 'Oppenheim's corrected ellipsoid' displayed an orientation to the galactic equator. The selection factors thus gave a result contrary to that which had been expected by Bourgeois and Cox.

The character of Oppenheim's ellipsoid indicates that the longest axis is perpendicular to the plane of the Galaxy, while the shortest axis is directed to the vertices of star-streaming. It should now be considered fully confirmed that the distribution of the major axes of cometary orbits reflects the main features of the motions of the stars in space and supports the hypothesis of the interstellar origin of comets. A few suggestions can be made in favour of that hypothesis:

(1) The motion of interstellar dust particles and gas molecules in the neighbourhood of the Sun depends on the galactic gravitational field and thus should follow the motions of stars in that part of space. Consequently, the motions of the particles reflect the phenomenon of star-streaming. Under the influence of solar radiation pressure a small particle decreases its velocity component in the direction of the Sun. When this velocity component has been reduced to zero, the particle will become a member of the solar system. Let $n$ be the ratio of light pressure to solar gravitational attraction, $\rho$ the distance of the particle from the Sun, $v$ the radial velocity component, $T$ and $\rho_{0}$ the time and the distance of the particle from the Sun when $v=0$. We then obtain, for example,

$$
\begin{aligned}
& \text { if } n=2, \rho=10000 \mathrm{AU}, v=1 \mathrm{~km} \mathrm{~s}^{-1} \text {; then } \\
& T=50000 \mathrm{yr}, \rho_{0}=1500 \mathrm{AU} ; \\
& \text { if } n=5, \rho=40000 \mathrm{AU}, v=3 \mathrm{~km} \mathrm{~s}^{-1} \text {; then } \\
& T=65000 \mathrm{yr}, \rho_{0}=720 \mathrm{AU} .
\end{aligned}
$$

Over comparatively short intervals of time, condensation takes place in the gas-anddust cloud in the Sun's vicinity; the probability of collisions between the particles is increased, leading to the formation of aggregations, and these would be too large to be affected by light pressure.

(2) In the opinion of many authors comet-like bodies exist in interstellar space in great quantities (Kepler, Russell, Lyttleton). According to Fehrenbach the total mass of interstellar meteorites inside a sphere of radius $r=10^{5} \mathrm{AU}$ amounts to $1 / 500$ the mass of the Sun, or the mass of $10^{15}$ comets. 
(3) These cosmic comets move along galactocentric orbits similar to those described by the stars.

(4) Assuming that the distance of the Sun from the centre of the Galaxy is $10^{4} \mathrm{pc}$, that its velocity is $285 \mathrm{~km} \mathrm{~s}^{-1}$, and that the mass of the Galaxy is $1.9 \times 10^{11} \mathrm{M}_{\odot}$, we find, according to Laplace's definition, that the radius of the Sun's sphere of action is $60000 \mathrm{AU}$. Comets entering this sphere of action from interstellar space may be captured by the Sun. It has now been established that capture is possible in the general problem of three bodies (Schmidt, 1951; Khil'mi, 1951; Sizova, 1952). Furthermore, it has long been known that under the influence of perturbations by Jupiter comets may be ejected from the planetary system; hence the reverse process, the capture of a cosmic comet by the solar system, is also possible. In accordance with the theory of probability most cases of capture occur at considerable distances from the Sun, so that comets moving along hyperbolic orbits will pass undetected at great distances from us. The only observable comets will be those captured in elliptical orbits of great eccentricity; comets with small orbital eccentricities will remain at great distances from the Sun and may in fact form the Oort cloud.

In summary the following conclusions may be inferred.

The main arguments against the interstellar origin of comets, namely the absence of hyperbolic cometary orbits (Strömgren) and the condition of zero relative velocity of the Sun and the comet (Schiaparelli) are not valid.

Studies of cometary statistics have detected the existence of comet-streaming similar to star-streaming. This phenomenon, as well as the determination of the solar apex from the distribution of the perihelia of cometary orbits (Tyror, Hurnik), are in favour of the hypothesis of interstellar origin.

As yet the mathematical aspects of the capture hypothesis have not been sufficiently developed. Specific initial conditions are required, and the process can hardly ensure a sufficient quantity of comets for the solar system.

Capture will be simplified if a decrease in the comet's heliocentric velocity takes place. Two possibilities should be taken into account: existence of a resisting medium or an increase in the mass of the comet. The first possibility should be rejected on the strength of the known data concerning interstellar space.

We may assume the existence of a spherical, uniform, gas-and-dust cloud surrounding the Sun at a distance of $100000 \mathrm{AU}$ and more. The particles in that cloud describe elliptical orbits of small eccentricity. For distances between 100000 and $230000 \mathrm{AU}$ only direct motion ensures stability (Chebotarev, 1964). The existence of such a cloud is proven by, among other things, the process of 'renewal' of comets as they replenish their supplies of gas and dust near aphelion. An interstellar comet entering the sphere on a hyperbolic orbit will start to increase its mass. The capture cross-section of the comet is given by $D^{2}=r_{0}^{2}\left(1+v_{c}^{2} / v_{r}^{2}\right)$, where $r_{0}$ is the radius of the comet, $v_{c}$ the escape velocity, and $v_{r}$ the relative velocity of the particle. During each astronomical unit of its passage through the gas-and-dust layer (density $\delta$ ) the comet accretes mass $\Delta m_{c}=$ $1.5 \times 10^{13} \pi r_{0}^{2} \delta \mathrm{g} \mathrm{cm}^{-3}$. Assuming the thickness of the layer to be $1000 \mathrm{AU}, r_{0}=10^{7} \mathrm{~cm}$, $\delta=10^{-18} \mathrm{~g} \mathrm{~cm}^{-3}, m_{c}=10^{17} \mathrm{~g}$, we find that the comet's relative mass accretion would be $10^{-5}$, which is not sufficient for our purposes. 
In order to decrease the comet's velocity it is necessary to assume that the cloud consists of a number of aggregations. These aggregations would accelerate the process of accretion and produce a comet consisting of a cosmic and a planetary component (Witkowski, 1968).

By analysing this hypothesis Piotrowski (1965) has shown that if the capture occurs at great distances from the Sun, it always leads

(1) to an increase in $1 / a$;

(2) to a decrease in $e$ for parabolic orbits;

(3) to a decrease in $e$ for hyperbolic retrograde orbits and most often to an increase in $e$ for direct, distinctly hyperbolic orbits; and generally;

(4) to an increase of the chance of discovery of interstellar comets with retrograde motion compared with comets moving along direct orbits.

Statements (3) and (4) are valid under the assumption of direct motions in the hypothetical cloud.

From a general point of view the modified hypothesis of capture may be brought into agreement with observational data. It ensures a constant supply of new comets, replacing the old ones which have disintegrated or have been thrown back into space.

Comets may be regarded as the visible links of a process of perpetual exchange of matter between the solar system and the rest of the universe. Comets transfer gas and dust from one region of our solar system to another, from the periphery to the central part of the system.

Finally I should like to mention an attempt at estimating the number of cosmic comets in the Galaxy. Our planetary system bears traces of an encounter with another planetary system several milliards of years ago. Pluto, and also the twin planets Uranus and Neptune, seem to speak in favour of such an encounter. Oort's cloud of comets itself may consist of debris following the close encounter of two planets from two different planetary systems. Accepting that one such encounter takes place once in four milliard years and that 10 interstellar comets enter our planetary system each year, we find that a sphere of radius $10^{5}$ AU contains $5 \times 10^{10}$ comets (the same order as that found by Oort for his cloud) and that the total number of cosmic comets in the Galaxy is about $5 \times 10^{22}$.

I have tried to give here arguments in favour of the interstellar origin of comets. Further work on the subject is necessary, and only the future will show which of the two hypotheses, the Laplacean or the Lagrangean, is correct.

\section{References}

Bondi, H. and Hoyle, F.: Monthly Notices Roy. Astron. Soc. 104, 273.

Bourgeois, P. and Cox, J.-F.: 1934, Bull. Astron. 8, 271 ; 9, 349.

Chebotarev, G. A.: 1964, Astron. Zh. 41, 983.

Galibina, I. V.: 1963, Byull. Inst. Teor. Astron. 9, 46.

Gething, P. J. D.: 1951, Monthly Notices Roy. Astron. Soc. 111, 468.

Hoffmeister, C.: 1951, Sterne 27, 189.

Hoffmeister, C.: 1952, Sterne 28, 229.

Hurnik, H.: 1959, Acta Astron. 9, 207.

Khil'mi, G. F.: 1951, Problema n Tel b Nebesnoj Mekhanike i Kosmogonii, Moscow. 
Kresák, L.: 1957, Contr. Astron. Obs. Skelnaté Pleso 2, 19.

Lyttleton, R. A.: 1948, Monthly Notices Roy. Astron. Soc. 108, 465.

McCrea, W. H.: 1953, in La Physique des Comètes, Liège, p. 337.

Oort, J. H.: 1950, Bull. Astron. Inst. Neth. 11, 91.

Oort, J. H.: 1951, Observatory 71, 129.

Oppenheim, S.: 1922, Astron. Nachr. 216, 47.

Oppenheim, S.: 1924, in Festschrift für H. v. Seeliger, Berlin.

Orlov, S. V.: 1939, Astron. Zh. 16, (1), 3.

Piotrowski, S. L.: 1965, Acta Astron. 15, 281.

Richter, N. B.: 1963, The Nature of Comets, Methuen, London.

Schmidt, O.: 1951, Izv. Akad. Nauk SSSR.

Sinding, E.: 1948, Publ. Mind. Medd. Kbh. Obs. No. 146.

Sizova, O. A.: 1952, Dokl. Akad. Nauk SSSR 86, 485.

Strömgren, E.: 1914, Publ. Mind. Medd. Kbh. Obs. No. 19.

Strömgren, E.: 1916, Astron. Nachr. 203, 17.

Svedstrup, A.: 1883, Astron. Nachr. 107, 113.

Tyror, J. G.: 1957, Monthly Notices Roy. Astron. Soc. 117, 370.

van Woerkom, A. J. J.: 1948, Bull. Astron. Inst. Neth. 10, 399.

Vsekhsvyatskij, S. K.: 1930, Monthly Notices Roy. Astron. Soc. 90, 706.

Vsekhsvyatskij, S. K.: 1931, Astron. Nachr. 243, 281.

Veskhsvyatskij, S. K.: 1952, Astron. Zh. 29, 63.

Witkowski, J. M.: 1953, Bull. Soc. Amis Sci. Lettres Poznań Ser. B 12, 205.

Witkowski, J. M.: 1968, Observatory 88, 27. 